\title{
is Research Square fand \\ Validity of two-dimensional analyses for the assessment of dynamic foot alignment during walking
}

Takasuke Miyazaki

Kagoshima Daigaku Igakubu

Masayuki Kawada

Kagoshima Daigaku Igakubu

Ryoji Kiyama ( $\square$ kiyama@health.nop.kagoshima-u.ac.jp )

Kagoshima University https://orcid.org/0000-0002-1819-9105

Kazunori Yone

Kagoshima Daigaku Igakubu

\section{Research}

Keywords: Motion capture, Tablet computer, Arch height, Foot posture index, Gait analysis

Posted Date: May 8th, 2020

DOI: https://doi.org/10.21203/rs.3.rs-27020/v1

License: (a) (i) This work is licensed under a Creative Commons Attribution 4.0 International License.

Read Full License 


\section{Abstract}

Background Several studies indicate that static foot posture bears a low relevance to dynamic foot alignment during walking. Thus, dynamic alignment assessment capable of being utilized in a clinical practice is required for an accurate estimation of foot function. This study aimed to examine the validity of two-dimensional analysis using a tablet computer for estimation of foot alignment during walking, by making a comparison with a motion capture system and static foot alignment screenings.

Methods Validity of two-dimensional (2D) analysis of foot alignment during walking using a tablet computer, by making a comparison with a motion capture system and static screenings in healthy subjects. Fourteen healthy males and 15 healthy females participated in this study. Arch height of the right foot while walking was measured using a tablet computer and a motion capture system simultaneously. Arch height at the mid-stance (Mst) and pre-swing (PSw) phase were calculated from kinematic data measured by the tablet computer and motion analysis system, respectively. Static foot alignment also was assessed through the use of a screening test including arch height index and foot posture index. To examine the validity of foot assessment using 2D analysis, we conducted correlation analysis between the arch height at Mst and PSw as measured by a tablet computer and a motion capture system. Meanwhile, FPI and AHI were used to test the relation to arch height at Mst as measured by a motion capture system.

Results Arch height, as measured by the motion capture system, showed a significant correlation with that measured using a tablet computer at Mst $(r=0.90, p<0.001)$ and PSw $(r=0.94, p<0.001)$, and arch height index $(r=0.50, p=0.005)$, especially the former. Meanwhile, foot posture index $(r=-0.34, p=$ 0.075 ) showed no relation to arch height as measured by the motion capture system.

Conclusion These results demonstrate the high validity of 2D analysis using a tablet computer for estimation of foot alignment during walking. Such gait analysis can be effective in assessing dynamic foot alignment in clinical practice.

\section{Background}

The foot and ankle contain a large number of bones and joints; it is the only part of human body that contacts the ground during standard locomotion, thus articulation within this region is important. While walking, the foot and ankle act to absorb much of the impact force at the early stance phase as well as to generate propulsion force during late stance [1-3]. In addition, foot dysfunction and malalignment is related to decreased function or injury throughout the whole lower limb [4-6]. Thus, foot alignment is usually assessed in physical therapy practice for lower limb injuries, Arch Height Index (AHI) and Foot Posture Index (FPI) are commonly used as screening tests [7-9]. These tests estimate static foot alignment during standing.

Previous studies report that $\mathrm{AHI}$, arch height during relaxed standing, are correlated with the trajectory of planter pressure during gait [10]. Meanwhile, foot malalignment including excessive foot supination relate 
to change in the alignment of the knee joint, resulting in increased load to lower limb joints under weightbearing conditions [11-13]. Thus, foot malalignment can be related to both lower leg injuries including medial tibial stress syndrome and Achilles tendon inflammation [4, 5], and also to knee joint injuries including patellofemoral joint pain [14-16].

However, several studies indicate that static foot posture bears a low relevance to dynamic foot alignment during walking, because of foot posture change due to ground reaction force and tibial motion $[17,18]$. Thus, in addition to static assessment, dynamic alignment assessment is required for an accurate estimation of foot function. Although foot alignment during gait has been analyzed using a three-dimensional motion capture system in previous studies [19-21], motion capture systems are not used widely in clinical practice because of the required time for measurement and processing, limitation of measurement space, and economic cost. In clinical practice, two-dimensional (2D) analysis using videos recorded using a video camera or tablet computer are usually utilized to assess gait function, in place of a motion capture system [22-24]. 2D analysis is easily measured anywhere, and feedback can be given to patients immediately. However, the validity of dynamic assessment of foot alignment using $2 \mathrm{D}$ analysis is unclear due to the lack of previous studies $[25,26]$. The aim of this study was to examine the validity of 2D analysis using a tablet computer for the estimation of foot alignment during walking, while making a comparison with a motion capture system and foot alignment screening tests. We hypothesized that foot alignment as measured by a tablet computer would be highly correlated with results obtained from a motion capture system, as opposed to static screening, including AHI and FPI.

\section{Methods}

\section{Subjects}

Fourteen healthy males (age, $24.9 \pm 3.2 \mathrm{y}$; height, $1.73 \pm 0.05 \mathrm{~m}$; weigh, $67.5 \pm 9.5 \mathrm{~kg}$ ) and 15 healthy females (age, $23.2 \pm 1.4 \mathrm{y}$; height, $1.60 \pm 0.04 \mathrm{~m}$; weight, $49.4 \pm 4.4 \mathrm{~kg}$ ) participated in this study; their right foot was measured. Subjects with orthopedics or neurological disorders were excluded. Informed consent was obtained from all participants before their inclusion in the study, and the ethics committee of the Faculty of Medicine, Kagoshima University approved the study protocol (ref no. 359).

\section{Procedure}

The relation between arch height during walking as measured by a tablet computer and motion capture system, and static foot alignments tests were estimated in order to test the validity of dynamic assessment using 2D analysis. Arch height of the right foot during walking was simultaneously measured using a tablet computer (iPad Air2, Apple, Inc., CA, USA) and motion analysis system consisting of 6 cameras (VICON MX3, Oxford Metrics, Oxford, UK) and two force plates (BP600400, OR67, AMTI Inc., MA, USA). Prior to gait measurement, reflective markers were attached to the lateral and medial epicondyle, the lateral and medial malleolus, the first and fifth heads of the metatarsal bone, the medial and dorsal point of the calcaneus, and the navicular, according to a previous study [21]. The tablet computer was placed vertically on the floor at the left side of the walkway and $1.3 \mathrm{~m}$ from the midline, so 
that it could capture the medial aspect of the foot (Fig. 1). One central stance phase during an $8 \mathrm{~m}$ comfortable walking gait was analyzed. The measurement was performed after a warm-up period, and the mean of 10 samples was adopted as the representative value. The sampling frequencies of the motion capture system and the tablet computer were $100 \mathrm{~Hz}$ and $120 \mathrm{~Hz}$, respectively.

Static foot posture was assessed using the arch height index (AHI) and foot posture index (FPI) during relaxed standing after several steps. AHI was the ratio calculated by dividing the dorsal arch height at $50 \%$ of total foot length, measured by height gage (VHK-15, Niigata Seiki Co, Ltd, Niigata, Japan), by the total foot length [9]. The FPI consisted of 6 components: talar head palpation, supra and infra lateral malleolar curvature, calcaneal frontal plane position, bulging in the region of the talo-navicular joint, height and congruence of the medial longitudinal arch, and abduction/adduction of the forefoot on the rearfoot [7]. Each component was scored on a scale ranging from -2 to +2 , and the total score ranged from -12 to +12 ; a low value indicated pronation, high values indicated supination.

\section{Data analysis}

Arch height at the mid-stance (Mst) phase, the moment when the right tibia is positioned vertically, and at the pre-swing (PSw) phase, the moment of heel strike of the opposite side, were obtained from kinematic data measured by the tablet computer and motion analysis system, respectively. In 2D analysis using the tablet computer, arch height was calculated as the distance between the navicular tuberosity and the baseline connecting to the medial aspect of the calcaneus and the first metatarsal head, and calculated as a percentage of the baseline using ImageJ (National Institute of Mental Health, MD, USA) by Windows PC (FMV-BIBLO NF/G50, Fujitsu, Kanagawa, Japan). In three-dimensional (3D) analysis, arch height was calculated as the distance between the navicular tuberosity and a plane consisting of the first metatarsal head, fifth metatarsal head, and the dorsal point of the calcaneus.

Pre-statistical analysis showed no sex difference in foot alignment, therefore we treated males and females as one group. Furthermore, we performed power analysis to estimate the validity of sample size by referring to a previous study, which reports that the correlation coefficient between 2D analysis and 3D analysis, and between static foot alignment and dynamic foot alignment, were 0.76 and 0.56 , respectively [25]. Thus, power analysis was performed using the $G *$ Power, $r=0.50, a=0.05$, and power $(1-\beta)=0.8$, indicated that the required sample size was 26 . Thus, we accepted that this study had a suitable sample size.

To examine the validity of foot assessment using 2D analysis, we conducted correlation analysis between the arch height at Mst and PSw as measured by a tablet computer and a motion capture system. Meanwhile, FPI and AHI were used to test the relation to arch height at Mst as measured by a motion capture system because of the similarity of their measurement posture. These relationships were analyzed using Pearson's correlation coefficient or Spearman's rank correlation coefficient after data were tested for normality using the Shapiro-Wilk test. All statistical analyses were performed using R (2.8.1) statistical software, and significance was set at $5 \%$. 


\section{Results}

Gait velocity was $1.32 \pm 0.14 \mathrm{~m} / \mathrm{s}$ (male, $1.35 \pm 0.16 \mathrm{~m} / \mathrm{s}$; female, $1.29 \pm 0.13 \mathrm{~m} / \mathrm{s}$ ), and step length was $0.67 \pm 0.06 \mathrm{~m}$ (male, $0.71 \pm 0.06 \mathrm{~m}$; female, $0.65 \pm 0.04 \mathrm{~m}$ ). Arch height as measured by the motion capture system decreased from 0 to $75 \%$, after which it increased during toe off (Fig. 2). Arch height ranged from $14.3 \pm 4.7$ to $24.9 \pm 5.1 \mathrm{~mm}$; it was $17.7 \pm 4.7 \mathrm{~mm}$ at MSt (25.4 $\pm 7.4 \%$ of the stance phase), and $15.0 \pm 4.7 \mathrm{~mm}$ at PSw $(82.0 \pm 2.1 \%$ of the stance phase $)$.

Arch height as measured by $2 \mathrm{D}$ was $11.0 \pm 2.8 \%$ at MSt, and $9.7 \pm 3.0 \%$ at PSw. With regard to foot alignment screening, $\mathrm{AHI}$ was $24.3 \pm 1.8 \%$ and FPI was $3.3 \pm 2.1$ points.

Arch height as measured using both the tablet computer and motion capture system were highly correlated [MSt, $r=0.90, p<0.001$ (Fig. 3a); PSw, $r=0.94, p<0.001$, (Fig. 3b)]. A significant correlation between foot alignment screening and the capture system was indicated in $\mathrm{AHI}[\mathrm{r}=0.50, p=0.005$ (Fig. 4a)], but not in FPI [rs $=-0.34, p=0.075$, (Fig. 4b)].

\section{Discussion}

We examined the validity of 2D analysis of foot alignment during walking using a tablet computer, by making a comparison with a motion capture system and static screenings in healthy subjects. The present study showed that arch height, as measured by a motion capture system, was significantly correlated with that measured by a tablet computer or AHI, especially the former. Meanwhile, FPI showed no relation to arch height as measured by the motion capture system. These results indicated the validity of dynamic assessment of foot alignment by using a tablet computer, and this was consistent with our hypothesis.

Arch height as measured by 3D was lowest during the later stance phase, which was similar to the findings of a previous study [21]. In the current study, arch height measured by the tablet computer showed a high correlation with the motion capture system. This relation was greater than that in the previous study. 2D analysis decreases accuracy for measuring a motion containing transverse rotation. However, the foot shows only slight transverse rotation during the stance phase of walking, because it is placed firmly on the floor. In addition, the tablet computer has high resolution in time and space. These factors contributed to the accuracy of the $2 \mathrm{D}$ analysis in this study.

With regard to foot alignment screening, AHI was $24.3 \pm 1.8(22.5-26.1) \mathrm{mm}$ and FPI was $3.3 \pm 2.1$ (1.25.4) points. Previous studies report standard values for each assessment [7, 9]; AHI ranged from 23.1 to $27.1 \mathrm{~mm}$ and $\mathrm{FPI}$ ranged from 0 to 5 points, where most participants showed a neutral foot alignment, with no excessive varus or valgus foot symptoms. In the correlation analysis, AHI was correlated with arch height, as measured by the motion capture system, but the correlation coefficient was lower than that measured by the tablet computer. Moreover, FPI were not correlated with arch height as measured by 
the motion capture system; this was consistent with a previous study [17]. The static screening estimates foot alignment during relaxed standing with bilateral limb support, thus, one half of total body weight was loaded on each foot. Dicharry [17] indicates that foot alignment during relaxed standing is influenced by gravity, but foot alignment during antigravitational activities including gait is influenced by muscle force and acceleration of the center of mass in addition to gravity. The maximum load on the foot during walking is almost $120 \%$ of body weight. Meanwhile, it is well known that motion of the shank affects alignment of the foot while the foot is placed on the floor, as a closed kinematic chain [27]. The difference in the load of the foot and the alignment of the shank between static standing and gait are the cause of a slight correlation between the static screenings and the arch height during gait.

To examine foot load, we focused on arch height at MSt and PSw. MSt is a single support phase when the full body weight loads to the unilateral lower limb; PSw is the end of the single support phase when arch height has decreased due to the ground reaction force acting on the forefoot. Malalignment of the foot becomes apparent at this time as opposed to relaxed standing, thus arch height at MSt and PSw is a relevant indicator reflecting foot alignment. Gait analysis obtained using a tablet computer could easily estimate arch height at these time points, therefore, such analysis is useful in clinical practice.

There were some limitations to this study. Since participants were healthy subjects, we made no analysis for varus and valgus foot deformities. In addition, we analyzed foot alignment only during walking, and not during sporting activities such as running or landing in which excessive force acts on the foot. Future investigations that address these issues are required for $2 \mathrm{D}$ analysis of foot alignment using a tablet computer in clinical practice. The present study contributes to the development of physical therapy for lower limb injuries.

\section{Conclusions}

In this study, we examined the validity of 2D analysis using a tablet computer for measurement of foot alignment during walking. Results showed that arch height, measured using a tablet computer, was highly correlated with measurements taken using a motion capture system while static foot alignment screening showed a lower correlation with arch height during walking. Consequently, 2D analysis using a tablet computer proved useful in the assessment of dynamic foot alignment in clinical practice.

\section{Abbreviations}

AHI: Arch Height Index; FPI: Foot Posture Index; 2D: two-dimensional; Mst: Mid-stance; PSw: Pre-swing; 3D: three-dimensional

\section{Declarations}

Ethics approval and consent to participate: The ethics committee of the Faculty of Medicine, Kagoshima University approved the study protocol (ref no. 359), and informed consent was obtained from all 
participants before their inclusion in the study.

Consent for publication: Not applicable.

Availability of data and materials: The datasets generated during the current study are available from the corresponding author on reasonable request.

Competing interests: There is no conflict of interest statement.

Funding: This research did not receive any specific grant from funding agencies in the public, commercial, or no profit sectors.

Authors' contributions: T.M. was responsible for study conceptualization and design, analysis and interpretation of data, and writing the manuscript. M.K. performed data collection and conducted the literature review. R.K. oversaw data collection and was responsible for data analysis. K.Y conducted the literature review. All authors contributed to and approved the final manuscript.

Acknowledgements: Not applicable.

\section{References}

1. Whittle MW. Generation and attenuation of transient impulsive forces beneath the foot: a review. Gait Posture. 1999;10:264-75.

2. Winter DA. Human blance and posture control during standing and walking. Gait Posture. 1995;3:193-214.

3. Stefanyshyn DJ, Nigg BM. Mechanical energy contribution of the metatarsophalangeal joint to running and sprinting. J Biomech. 1997;30:1081-5.

4. Dowling GJ, Murley GS, Munteanu SE, Smith MMF, Neal BS, Griffiths IB, et al. Dynamic foot function as a risk factor for lower limb overuse injury: a systematic review. J Foot Ankle Res. 2014;7:53.

5. Neal BS, Griffiths IB, Dowling GJ, Murley GS, Munteanu SE, Franettovich Smith MM, et al. Foot posture as a risk factor for lower limb overuse injury: a systematic review and meta-analysis. J Foot Ankle Res. 2014;7:55.

6. Tong JWK, Kong PW. Association between foot type and lower extremity injuries: Systematic literature review with meta-analysis. J Orthop Sports Phys Ther. 2013;43:700-14.

7. Redmond AC, Crosbie J, Ouvrier RA. Development and validation of a novel rating system for scoring standing foot posture: the Foot Posture Index. Clin Biomech (Bristol Avon). 2006;21:89-98.

8. Carvalho BKG de, Penha PJ, Penha NLJ, Andrade RM, Ribeiro AP, João SMA. The influence of gender and body mass index on the FPI-6 evaluated foot posture of 10- to 14-year-old school children in São Paulo, Brazil: A cross-sectional study. J Foot Ankle Res Journal of Foot Ankle Research. 2017;10:17. 
9. Butler RJ, Hillstrom H, Song J, Richards CJ, Davis IS. Arch height index measurement system: establishment of reliability and normative values. J Am Podiatr Med Assoc. 2008;98:102-6.

10. Teyhen DS, Stoltenberg BE, Collinsworth KM, Giesel CL, Williams DG, Kardouni CH, et al. Dynamic plantar pressure parameters associated with static arch height index during gait. Clin Biomech (Bristol Avon). 2009;24:391-6.

11. Hunt AE, Smith RM. Mechanics and control of the flat versus normal foot during the stance phase of walking. Clin Biomech (Bristol Avon). 2004;19:391-7.

12. Hösl M, Böhm H, Multerer C, Döderlein L. Does excessive flatfoot deformity affect function? A comparison between symptomatic and asymptomatic flatfeet using the Oxford Foot Model. Gait Posture. 2014;39:23-8.

13. Resende RA, Kirkwood RN, Deluzio KJ, Hassan EA, Fonseca ST. Ipsilateral and contralateral foot pronation affect lower limb and trunk biomechanics of individuals with knee osteoarthritis during gait. Clin Biomech (Bristol Avon). 2016;34:30-7.

14. Barton CJ, Munteanu SE, Menz HB, Crossley KM. The efficacy of foot orthoses in the treatment of individuals with patellofemoral pain syndrome: a systematic review. Sports Med. 2010;40:377-95.

15. Reilly K, Barker K, Shamley D, Newman M, Oskrochi GR, Sandall S. The role of foot and ankle assessment of patients with lower limb osteoarthritis. Physiotherapy. 2009;95:164-9.

16. Levinger P, Menz HB, Fotoohabadi MR, Feller JA, Bartlett JR, Bergman NR. Foot posture in people with medial compartment knee osteoarthritis. J Foot Ankle Res. 2010;3:29.

17. Dicharry JM, Franz JR, Della Croce U, Wilder RP, Riley PO, Kerrigan DC. Differences in static and dynamic measures in evaluation of talonavicular mobility in gait. J Orthop Sports Phys Ther. 2009;39:628-34.

18. Paterson KL, Clark RA, Mullins A, Bryant AL, Mentiplay BF. Predicting Dynamic Foot Function From Static Foot Posture: Comparison Between Visual Assessment, Motion Analysis, and a Commercially Available Depth Camera. J Orthop Sports Phys Ther. 2015;45:789-98.

19. Buldt AK, Levinger P, Murley GS, Menz HB, Nester CJ, Landorf KB. Foot posture is associated with kinematics of the foot during gait: A comparison of normal, planus and cavus feet. Gait Posture. 2015;42:42-8.

20. Leardini A, Benedetti MG, Berti L, Bettinelli D, Nativo R, Giannini S. Rear-foot, mid-foot and fore-foot motion during the stance phase of gait. Gait Posture. 2007;25:453-62.

21. Simon J, Doederlein L, Mclntosh AS, Metaxiotis D, Bock HG, Wolf SI. The Heidelberg foot measurement method: development, description and assessment. Gait Posture. 2006;23:411-24.

22. Brunnekreef JJ, Van Uden CJT, Van Moorsel S, Kooloos JGM. Reliability of videotaped observational gait analysis in patients with orthopedic impairments. BMC Musculoskelet Disord. 2005;6:1-9.

23. Lo GH, Harvey WF, McAlindon TE. Associations of varus thrust and alignment with pain in knee osteoarthritis. Arthritis Rheum. 2012;64:2252-9. 
24. Taş S, Güneri S, Kaymak B, Erden Z. A comparison of results of 3-dimensional gait analysis and observational gait analysis in patients with knee osteoarthritis. Acta Orthop Traumatol Turc. 2015;49:151-9.

25. Bencke J, Christiansen D, Jensen K, Okholm A, Sonne-Holm S, Bandholm T. Measuring medial longitudinal arch deformation during gait. A reliability study. Gait Posture. 2012;35:400-4.

26. McPoil TG, Cornwall MW, Vicenzino B, Teyhen DS, Molloy JM, Christie DS, et al. Effect of using truncated versus total foot length to calculate the arch height ratio. Foot (Edinb). 2008;18:220-7.

27. Reischl SF, Powers CM, Rao S, Perry J. Relationship between foot pronation and rotation of the tibia and femur during walking. Foot Ankle Int. 1999;20:513-20.

\section{Figures}

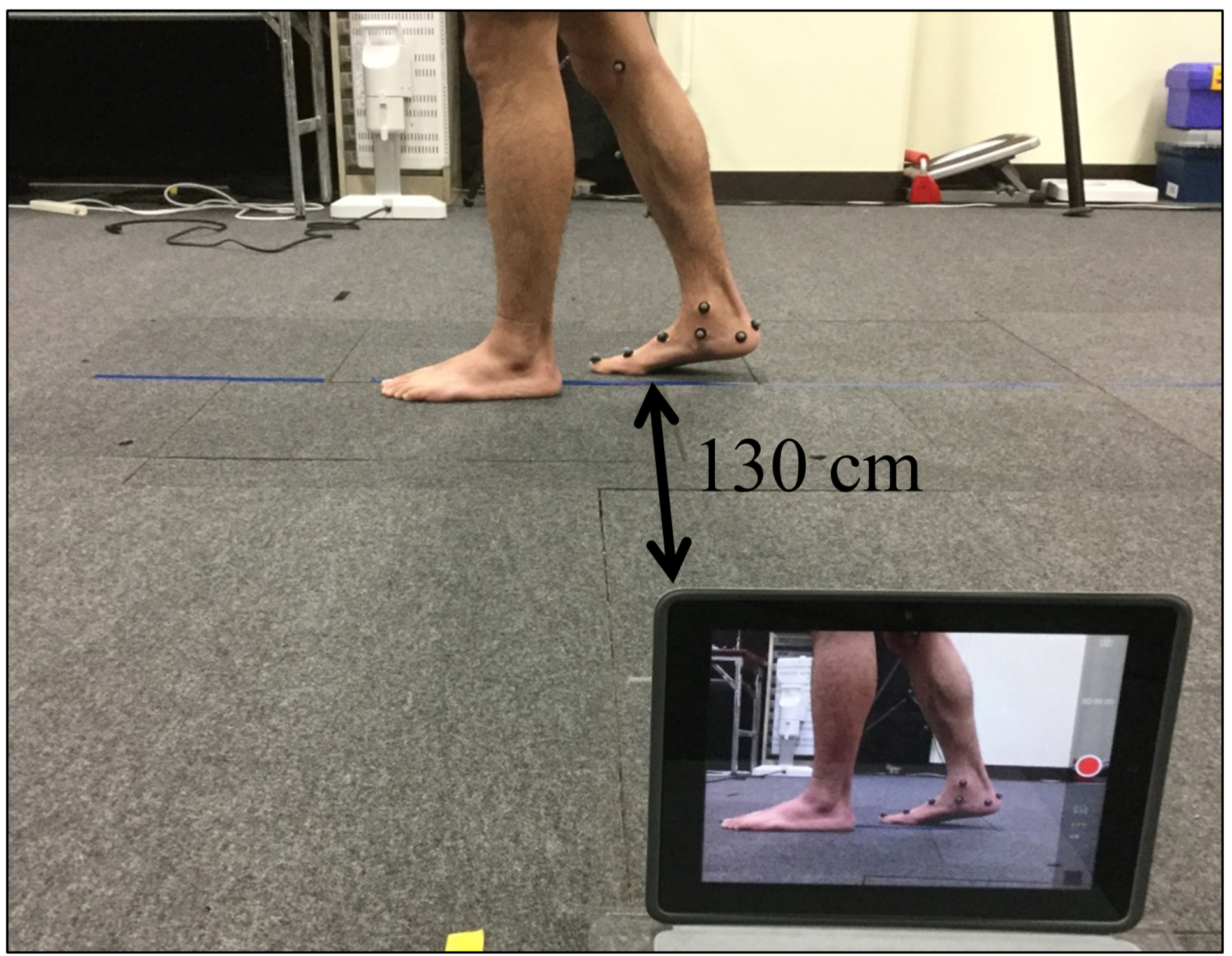

Figure 1 
Measurement of foot alignment during walking using a tablet computer and motion capture systems. The tablet computer was placed vertically on the floor at the left side of the walkway and $1.3 \mathrm{~m}$ from the midline, due to capture the medial aspect of the foot.

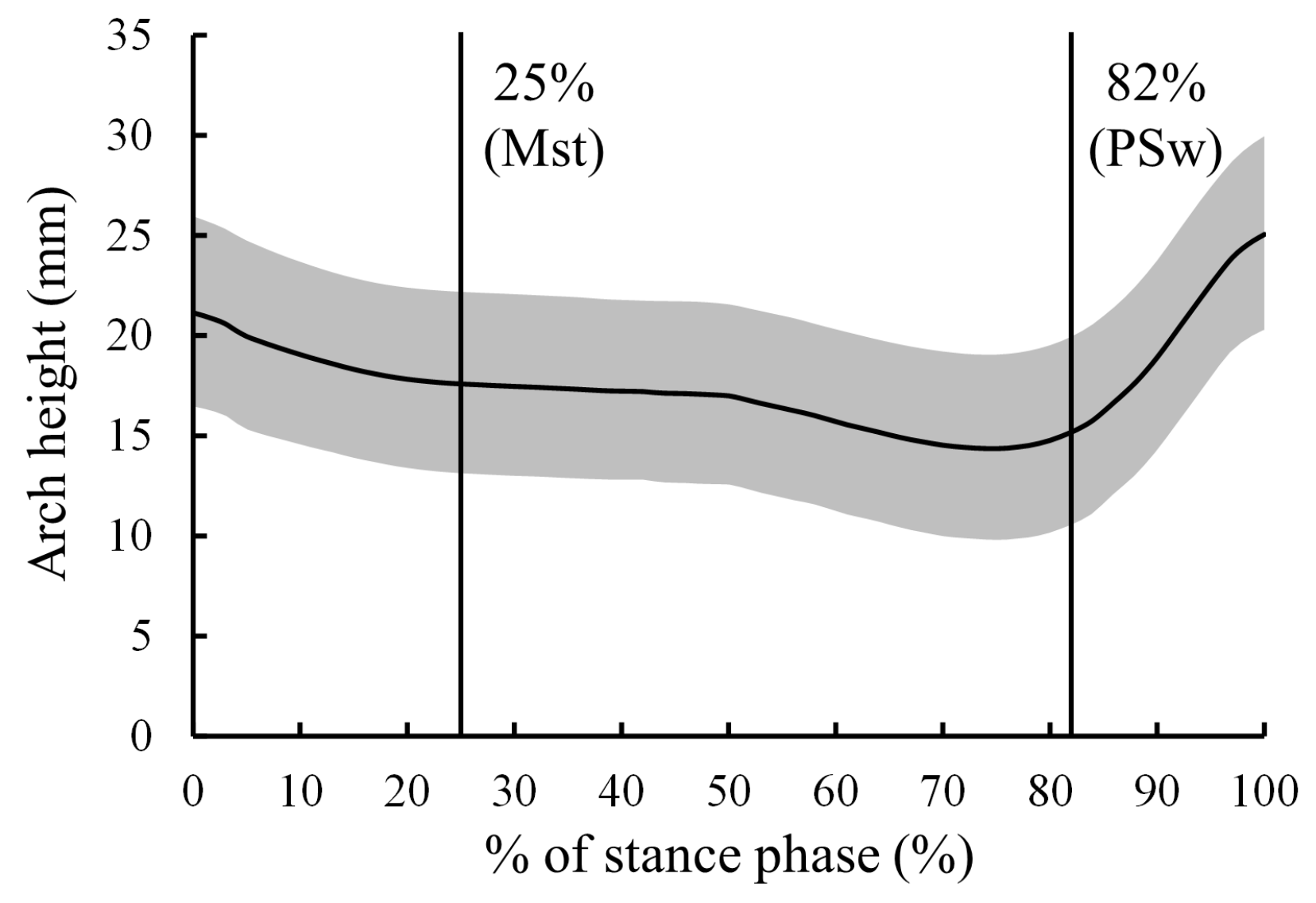

Figure 2

Ensemble average of arch height during the stance phase of all participants. The solid line indicates the mean value, and the gray area indicates standard deviation. The vertical lines indicate the mid-stance (MSt) and the pre-swing (PSw) phases. Arch height was lowest at $75 \%$ of the stance phase. 


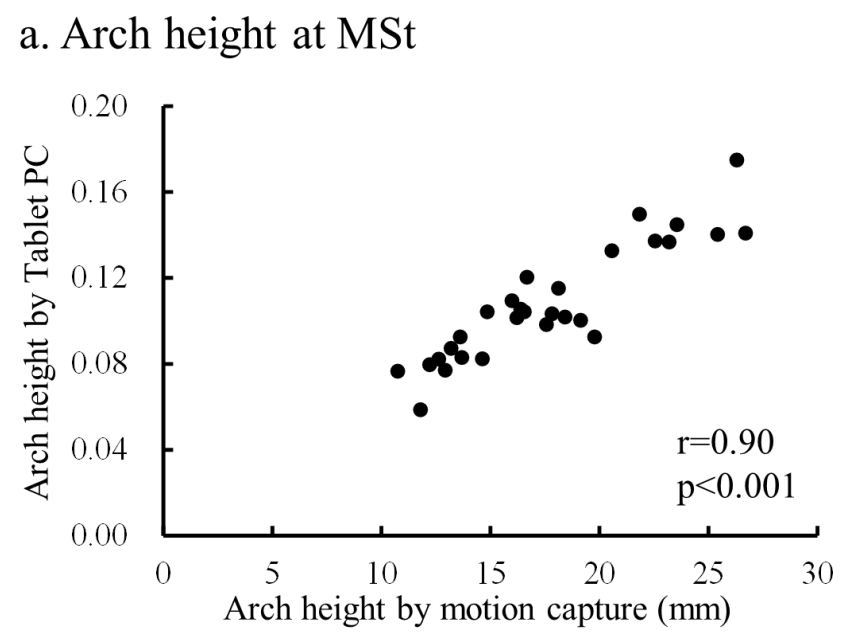

b. Arch height at PSw

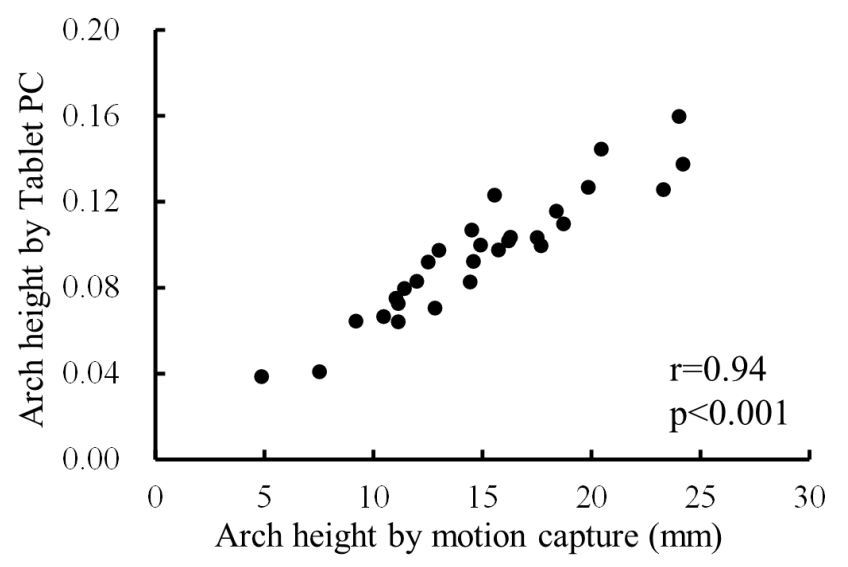

Figure 3

Scatter plot of arch height as measured by 3D motion capture and a tablet computer. These values were closely correlated with each other during the mid-stance (MSt) and pre-swing (PSw) phases.

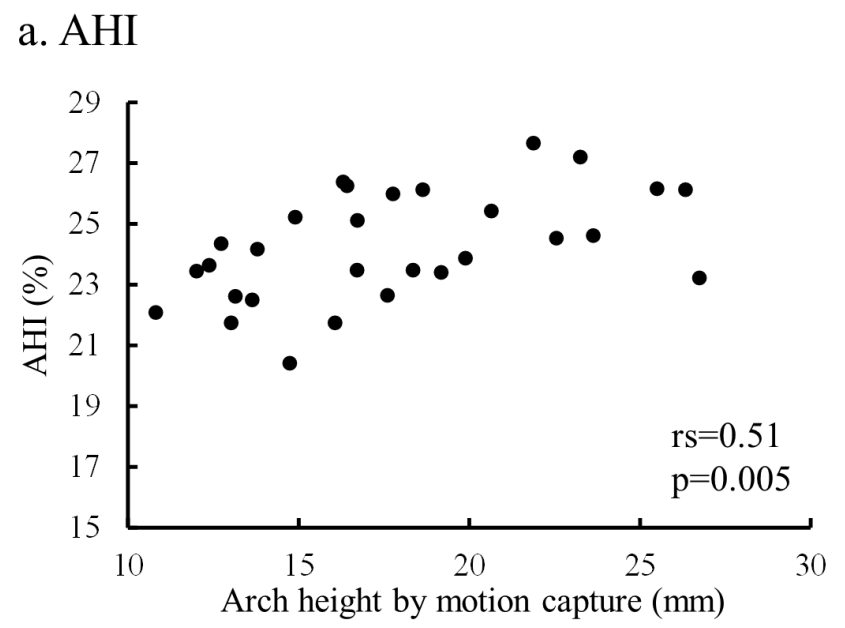

\section{b. FPI}

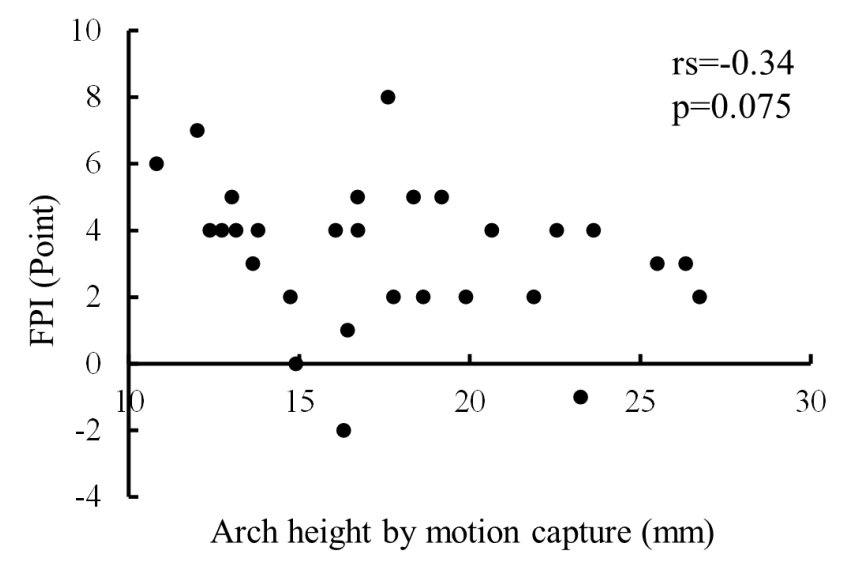

\section{Figure 4}

Scatter plot of foot alignment as measured using a 3D motion capture system vs. static foot alignment screening. A significant relationship was shown in arch height index (AHI) but not in the foot posture index (FPI). 